\title{
Quarkonium dynamics inside the Quark-Gluon Plasma using open quantum systems
}

\author{
Stéphane Delorme ${ }^{1, *}$, Thierry Gousset ${ }^{1}$, Roland $\mathrm{Katz}^{1}$, and Pol-Bernard Gossiaux ${ }^{1}$ \\ ${ }^{1}$ SUBATECH, Université de Nantes, IMT Atlantique, IN2P3/CNRS, 4 rue Alfred Kastler, 44307 Nantes \\ cedex 3, France
}

\begin{abstract}
In recent years, a significant theoretical effort has been made towards a dynamical description of quarkonia inside the Quark-Gluon Plasma (QGP), using the open quantum systems formalism. In this framework, one can get a real-time description of a quantum system (here the quarkonium) in interaction with a thermal bath (the QGP) by integrating out the bath degrees of freedom and studying the system reduced density matrix. We investigate the real-time dynamics of a correlated heavy quark-antiquark pair inside the QGP using a quantum master equation previously derived from first QCD principles in [4]. The full equation is directly resolved in $1 \mathrm{D}$ to lessen computing costs and is used for the first time to gain insight on the dynamics in both a static and evolving medium following a Björken-like temperature evolution.
\end{abstract}

\section{Quarkonium phenomenology}

At non-zero temperatures, the interaction between the heavy quark and antiquark of the pair is modified due to the presence of other color charges. As temperature increases, the density of color charges also increases, leading to stronger screening of the interaction and melting of the pairs at high temperature.[1] This screening is encoded in the real part V of a complex potential, which depends on both the distance between the quark and antiquark but also on the temperature [2]. The bound quark and antiquark system can also scatter on partons present in the medium, which may also lead to the pair dissociation. These collisional effects can be described by adding an imaginary part $\mathrm{W}$ to the in-medium quark-antiquark potential $\mathrm{V}$, which also depends on the temperature and relative distance between the quark and antiquark. The combination of the screening of the interaction and the collisions in the medium leads to quarkonia suppression. As the energy of the collisions increases, one would expect stronger suppression of quarkonium states. However, experimental measurements at RHIC and LHC showed a more complicated pattern, where the $J / \Psi$ suppression was much smaller than expected for some kinetics [3]. This partial disappearance of suppression is thought to be caused by recombination of initially uncorrelated charm and anticharm quarks inside the medium during the evolution and at the hadronization. This effect is also crucial for phenomenology, as there are a high number of $c$ and $\bar{c}$ quarks in the medium. It is quite clear that a quantum treatment of the real-time dynamics of heavy quarkonia is needed to properly treat both suppression and recombination. In order to do so, one can use the open quantum system formalism. In that framework, one can treat the dynamics of a system composed by a

\footnotetext{
*e-mail: delorme@ subatech.in2p3.fr
} 
quantum subsystem (a quarkonium) in interaction with its environment (the QGP). Only the quarkonium dynamics is of interest, so the environment degrees of freedom are traced out to obtain a quantum master equation describing the real-time quarkonium dynamics.

\section{Quantum master equation}

The present analysis is based on the work of Blaizot \& Escobedo [4]. Assuming a weak coupling between the heavy quarks and the plasma constituents and focusing on the quantum brownian motion regime (valid at high temperatures $T>>200 \mathrm{MeV}$ ), they derived coupled quantum master equations, taking into account color degrees of freedom:

$$
\frac{d}{d t}\left(\begin{array}{l}
\mathcal{D}_{s} \\
\mathcal{D}_{o}
\end{array}\right)=\mathcal{L}\left(\begin{array}{l}
\mathcal{D}_{s}\left(\mathbf{s}, \mathbf{s}^{\prime}, t\right) \\
\mathcal{D}_{o}\left(\mathbf{s}, \mathbf{s}^{\prime}, t\right)
\end{array}\right),
$$

where $\mathcal{D}_{s}$ and $\mathcal{D}_{o}$ are respectively the singlet and octet density matrices. $\mathbf{s}$ and $\mathbf{s}^{\prime}$ are respectively the distance between the quark and the antiquark and the conjugated variable. The $\mathcal{L}$ operator can be decomposed in the following way:

$$
\mathcal{L}=\left(\begin{array}{ll}
\mathcal{L}_{s S} & \mathcal{L}_{s o} \\
\mathcal{L}_{o s} & \mathcal{L}_{o o}
\end{array}\right),
$$

with the various $\mathcal{L}_{s s}, \mathcal{L}_{s o}, \mathcal{L}_{o s}$ and $\mathcal{L}_{o o}$ operators describing transitions between color states. We can also write $\mathcal{L}$ (and the other operators) as $\mathcal{L}=\mathcal{L}_{0}+\mathcal{L}_{1}+\mathcal{L}_{2}+\mathcal{L}_{3} . \mathcal{L}_{0}$ describes the free motion of the pair, $\mathcal{L}_{1}$ describes static screening (involving the real part of the potential V), $\mathcal{L}_{2}$ describes the fluctuations in the medium (involving the imaginary part of the potential $\mathrm{W}$ ) and $\mathcal{L}_{3}$ describes dissipative effects in the medium (involving derivatives of $\mathrm{W}$ ). In their approach, Blaizot \& Escobedo implemented a semi-classical approximation of their equations, resulting in Langevin equations. However, their equations break positivity at the $\mathcal{L}_{3}$ level, which is a core property of well-defined quantum master equations. We extended their equations ${ }^{1}$, modifying the $\mathcal{L}_{3}$ operator and adding a higher-order operator $\mathcal{L}_{4}$. With the new equations, positivity is preserved and the master equation is well-defined. The equations were then resolved in one dimension to reduce the computing cost while still being able to explore the dynamics.

\section{One-dimensionnal real-time dynamics of a heavy quarkonium in the Quark-Gluon Plasma}

\subsection{Fixed temperature case}

We consider a quark-gluon plasma with fixed temperature and take as the initial state the 1Slike eigenstate obtained from the vacuum potential V. Figure 1 shows the instantaneous projections of the singlet density matrix $\mathcal{D}_{s}$ on the first three eigenstates obtained from the vacuum potential V for $T=200,300$ and $400 \mathrm{MeV}$. The evolution starts with a transient phase where transitions from the initial 1S-like state occur, before the equilibration of the populations. At later times, all three eigenstates decay with the same rate (except for $T=200 \mathrm{MeV}$ ) but the populations do not follow a Maxwell-Boltzmann distribution at the considered temperatures. The whole evolution is characterized by the interplay between binding, diffusion and transition between states. Note that from $T=280 \mathrm{MeV}$, only the 1S-like state is bound with the potential used.

\footnotetext{
${ }^{1}$ Following a procedure described in Appendix B of [4].
} 

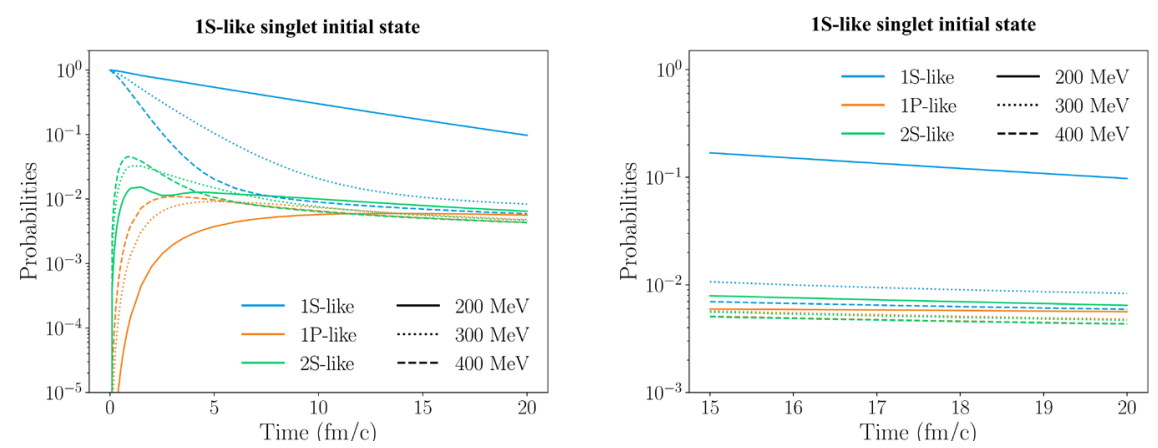

Figure 1. Left panel: Evolution over time of the vacuum eigenstates populations for a temperature $T=200,300$ and $400 \mathrm{MeV}$. Right panel: Zoom on the late-time evolution.

The analysis of the singlet density matrix $\mathcal{D}_{s}$ shows (see figure 2 ) the progressive delocalization of the initial state along the $s=s^{\prime}$ axis. Two components are observed: 1) a remnant of the initial state is present around $s=s^{\prime}=0 \mathrm{fm}$ and 2) a semi-classical-like component on the rest of the $s=s^{\prime}$ axis. The width of the distribution along the $s=-s^{\prime}$ for this component is quite small, indicating that strong decoherence effects occured during the evolution of the system.

$$
\mathcal{D}_{s} \quad \mathrm{~T}=300 \mathrm{MeV}
$$
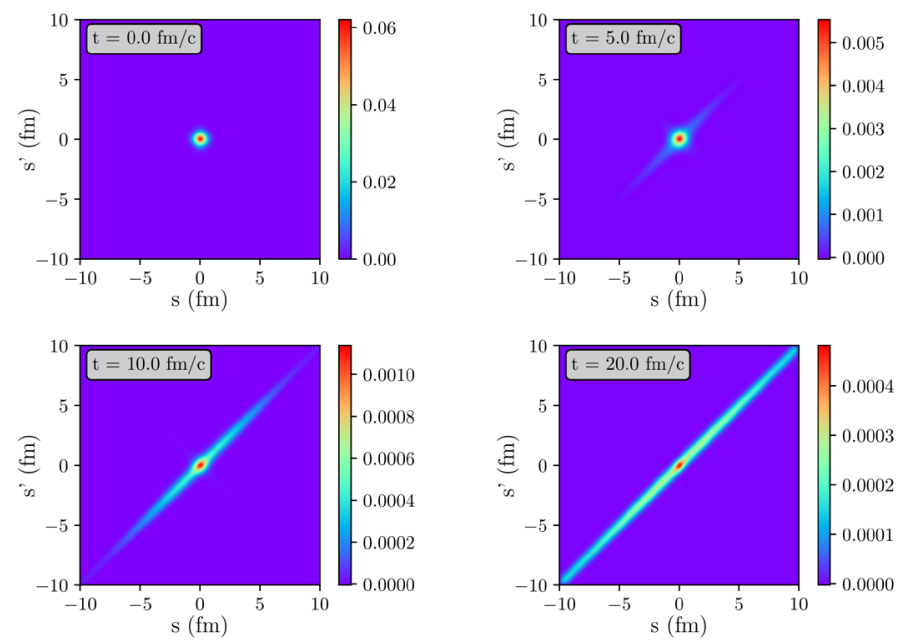

Figure 2. Evolution of the singlet density matrix $\mathcal{D}_{s}$ over time for a temperature $T=300 \mathrm{MeV}$.

\subsection{Cooling medium case}

To perform this study, we adopt a medium-temperature evolution following Björken's model [5], $T(t)=T_{0}\left(\frac{t_{0}}{t_{0}+t}\right)^{1 / 3}$, where $T_{0}$ is the medium initial temperature and $t_{0}$ the initial time taken 
to be equal to $1 \mathrm{fm} / \mathrm{c}$. We consider the case of a QGP medium with an initial temperature $T_{0}=400 \mathrm{MeV}$ and an initial 1S-like singlet state. We perform the instantaneous projection of the singlet density matrix $\mathcal{D}_{s}$ on the eigenstates obtained from the vacuum potential $\mathrm{V}$. The results are presented in figure 3 . We observe the creation at intermediate times of excited singlet bound states and the progressive re-equilibration of the populations. After around $8 \mathrm{fm} / \mathrm{c}$, the temperature of the medium gets below $T=200 \mathrm{MeV}$, which is a limit where the model may not be valid anymore as the quantum brownian motion assumptions are not realized anymore.

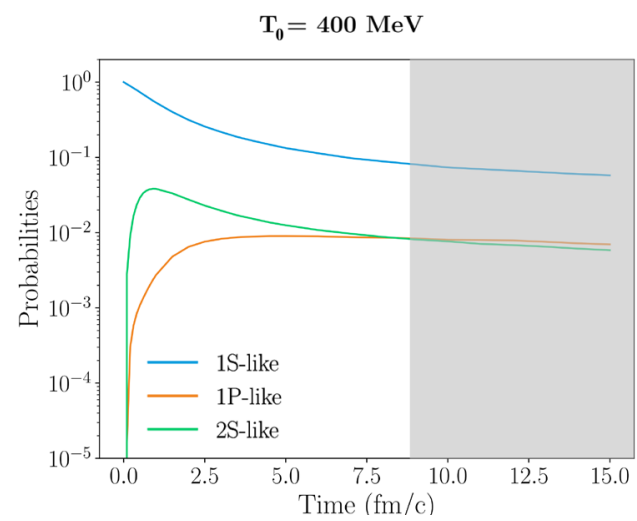

Figure 3. Evolution over time of the vacuum eigenstates populations from an initial temperature $T_{0}=$ $400 \mathrm{MeV}$. The grey band indicates the range in time where $T(t)<200 \mathrm{MeV}$.

\section{Conclusion}

We presented results on real-time quarkonium dynamics in the quark-gluon plasma described by new coupled quantum master equations. The equations involve transitions between color states as well as dissipative effects, and were directly resolved numerically for the first time in one dimension. The evolution is the result of the interplay between binding effects, diffusive effects and transitions between states. The analysis of the density matrix showed the delocalization of the initial state along a specific direction in the $\left(s, s^{\prime}\right)$ space and exhibited a semi-classical-like behaviour. More details on the model and updated results will be presented in an upcoming publication.

\section{Acknowledgements}

The authors thank Jean-Paul Blaizot and Alexander Rothkopf for valuable discussions. S.D. is supported by CNRS and Région Pays de la Loire.

\section{References}

[1] T. Matsui, H. Satz, Phys. Lett. B 178, 416 (1986)

[2] D. Lafferty, A. Rothkopf, Phys. Rev. D, 101 (2020)

[3] ALICE collaboration, Phys. Letter. B., 766, 212-224 (2017)

[4] J.P. Blaizot, M.A. Escobedo, J. High Energ Phys. 2018, 34 (2018)

[5] J.D. Björken, Phys. Rev. D, 27, 140-151 (1983) 